\title{
PUBLIC POLICY, MITIGATION AND ADAPTATION TO CLIMATE CHANGE IN SOUTH AMERICA
}

\author{
Msc.Rafael D'Almeida Martins \\ Doutorando do Programa em Pós-Graduação em Ambiente \& Sociedade - UNICAMP \\ Rua dos Flamboyants, 155 - CEP:13083-867 - Campinas (SP) - Brasil
}

Fone (19) 3521-5103 - rafael@cepps.org.br

RESENHA DE: DIAS, Pedro Leite da Silva; RIBEIRO, Wagner da Costa; NETO, João Lima Sant'Anna; ZULLO JR., Jurandir. Public Policy, Mitigation and Adaptation to Climate Change in South America. São Paulo: Instituto de Estudos Avançados; Universidade de São Paulo (IEA/ USP), 2009, 280 p. (http://www.iea.usp.br/cienciasambientais/climatechangeandsouthamerica.pdf)

A divulgação do quarto relatório do Painel Intergovernamental sobre Mudanças Climáticas (IPCC AR4 em inglês) no ano de 2007 consolidou o tema das mudanças ambientais globais na agenda internacional ao afirmar com alto grau de confiança que o aquecimento do planeta seria resultado, também, de atividades humanas. Tratou-se de afirmação contundente e hoje o tema tem presença garantida na mídia diária e o engajamento de comunidades políticas, empresariais e científicas que buscam articulação e coordenação de esforços diversos como estratégia para enfrentar o maior desafio do séc. XXI (GIDDENS, 2009).

Entretanto, a complexidade do t ema e a magnitude das adaptações necessárias para enfrentá-lo em termos de mudanças em padrões de produção e consumo, matriz energética e marcos institucionais, entre tantas outras, gera um infinito número de questões a serem discutidas e analisadas. O tema também é cercado de grande incerteza que mesmo com o avanço rápido da ciência do clima, permanecem lacunas importantes na compreensão e na modelagem de sistemas biogeofísicos da Terra. Mesmo assim, nos últimos anos, recentes evidências foram coletadas e analisadas e serviram de subsídio para que novas projeções fossem feitas, combinadas a avanços na capacidade de modelagem do sistema climático global (FÜSSEL, 2008). Esses estudos reforçam o entendimento de maioria esmagadora da comunidade científica que acredita na contribuição humana para mudança climática (SOLOMON et al., 2007; ZHANG et al., 2007; WILLETT et al., 2007; RAHMSTORF et al., 2007). Assim, como a incerteza é inerente ao processo, não é possível esperar mais tempo para adotar medidas que atenuem os prováveis impactos gerados pelo aquecimento global, sejam elas de mitigação ou de adaptação.

A dimensão, amplitude e extensão de boa parte dessas mudanças dependem da capacidade de mitigação, ou seja, da redução das emissões de gases de efeito estufa (GEE), matéria que vem sendo tratada no âmbito das negociações internacionais, em especial na Convenção-Quadro das Nações Unidas sobre a Mudança do Clima (UNFCCC em inglês). Tais negociações envolvem diversos interesses e avançam de forma lenta e gradual, muito aquém das necessidades defendidas pelos cientistas e pelos ambientalistas.

Do outro lado, como alguns impactos são inevitáveis, uma vez que mesmo com enormes esforços de mitigação (muito improváveis dado o panorama atual de negociação), o aquecimento global deverá ocorrer por várias décadas dado o nível de concentração de GEE na atmosfera. Dessa forma, ações de adaptação também se colocam como um imperativo a ser perseguido e implementado (NOBRE, 2007). Ribeiro (2008) defende a adoção do princípio da precaução como forma de acelerar medidas que possam atenuar as implicações causadas pelas mudanças globais.

No caso brasileiro, a situação é preocupante. O país tem pouca tradição em abordagens interdisciplinares que busquem avaliar os impactos das mudanças climáticas em ecossistemas, áreas rurais e urbanas (HOGAN, 2007; NOBRE, 2007). O envolvimento do país na pesquisa sobre as dimensões humanas da mudança climática é ainda frágil e busca consolidação (MARTINS; 
FERREIRA, 2009). Todavia iniciativas recentes mostram a vulnerabilidade de diferentes sistemas sócio-ecológicos brasileiros às mudanças climáticas . Soma-se a isso o contexto socioeconômico, que apesar da relativa melhora dos últimos anos, ainda apresenta parcela importante da população vivendo em situação de pobreza e precariedade, além do Brasil ser altamente dependente de recursos naturais e de sua biodiversidade (NOBRE, 2007). Faz-se necessário, assim, um envolvimento cada vez maior das ciências brasileiras para informar e pautar políticos, comunidades e setor privado das ameaças e oportunidades que estão colocadas.

Com base nesse esforço intelectual e científico necessário, o livro "Public Policy, Mitigation and Adaptation to Climate Change in South America" é uma contribuição importante nessa direção. A obra organizada pelos professores Pedro Leite da Silva Dias, Wagner da Costa Ribeiro, João Lima Sant'Anna Neto e Jurandir Zullo Jr. é resultado da III Conferência sobre Mudanças Globais na América do Sul, que aconteceu em Novembro de 2007 na cidade de São Paulo. O livro agrega contribuições de participantes de mesas redondas, bem como os artigos que foram reconhecidos pelo seu mérito científico em diferentes áreas como relações internacionais e políticas públicas, mitigação, adaptação e suas conseqüências para os sistemas naturais e ecológicos que compõem as quatro partes do livro publicado pelo Institudo de Estudos Avançados da Universidade de São Paulo (IEA/USP).

No primeiro capítulo, após breve introdução, Viola discute as perspectivas de um acordo internacional pós-Kyoto a partir dos perfis dos grandes emissores de GEE, com especial atenção a China, Estados Unidos, União Européia, Japão, Índia, Brasil e Rússia. O autor traça três diferentes cenários que poderão acontecer na construção da segurança climática global. O primeiro seria um cenário hobbesiano, pessimista por natureza; o segundo aponta para um Protocolo de Kyoto intensificado de caráter moderado e o terceiro seria um grande acordo global altamente otimista. Cada cenário é discutido em detalhes incluindo possíveis posicionamentos de cada nação, bem como mudanças de conjunturas nacionais e regionais no âmbito sul-americano.

O segundo capítulo discute possíveis estratégias e políticas que poderão ser adotadas para mitigar as emissões de GEE dando especial atenção à distribuição dos custos envolvidos para adoção dessas estratégias. Girardin, do Consejo Nacional de Investigaciones Científicas y Técnicas da Argentina, apresenta paradoxo já bastante explorado na literatura internacional (por exemplo, ver ADGER et al., 2006). Países em desenvolvimento contribuíram menos com o problema, porém sofrerão mais com os impactos. Assim, para esses países, assistência e financiamento para adaptação é fundamental e coloca-se como uma prioridade. Todavia, grande parte dos recursos disponíveis até o momento serve para interesses de mitigação, tendo como foco países em desenvolvimento de economias avançadas como China e Índia (MOG et al., 2009).

Pinguelli Rosa, no terceiro capítulo, aborda o processo de construção do Plano Nacional de Mudança do Clima no Brasil. No artigo, o autor apresenta propostas distribuídas em três eixos estratégicos: mitigação, adaptação e vulnerabilidade e ações transversais. Tais propostas foram discutidas por vários ministérios e setores da sociedade civil no âmbito do Comitê Interministerial sobre Mudança do Clima (CIM), órgão de caráter permanente composto por 16 ministérios e pelo Fórum Brasileiro de Mudanças Climáticas. Este comitê foi responsável por alinhar as diferentes iniciativas do governo, e, em especial, orientar a elaboração e implementação da Política Nacional e do Plano Nacional sobre Mudança do Clima (PNMC). Cabe destacar que essa política (Lei 12.187) foi sancionada pelo Presidente da República em 29 de Dezembro de 2009, consolidando o processo descrito no artigo.

No último artigo da primeira parte do livro, Cunha et al. apresentam uma iniciativa subnacional de governança climática no nível local a partir da experiência do Estado de São Paulo. Os autores destacam que na ausência de acordos mais audaciosos no plano internacional, diversos governos locais e sub-nacionais tiveram a liderança de implementar legislações que adotam metas de corte de emissões de GEE. A lei paulista recebeu sanção do governador em 9 de Novembro de 
2009 e demonstra que a governança do clima envolve diversos atores e não somente os tradicionais governos nacionais dos países envolvidos deixando claro que o desafio da mudança climática é multinível e multiescalar.

A segunda parte, intitulada Mitigação, inicia-se com artigo de Carloni et al. fazendo uma avaliação dos projetos brasileiros aprovados de Mecanismo de Desenvolvimento Limpo (MDL). O MDL é um dos mecanismos do Protocolo de Kyoto e o estudo mostra que existe potencial para vários setores da economia nacional adotar projetos dessa natureza. Atualmente, os projetos mais comuns estão restritos às áreas de energia e resíduos sólidos, em especial aterros sanitários. Porém, setores como agronegócios e florestas apresentam grande potencial, apesar de algumas barreiras administrativas e metodológicas que precisam ser enfrentadas. Em outro capítulo, Gamba e Ribeiro analisam a implementação de projeto de MDL buscando co-geração de energia no município de São Joaquim da Barra, nordeste do Estado de São Paulo. O projeto busca aumentar eficiência no processo de co-geração de energia a partir do bagaço da cana de açúcar. Os autores identificam potencial de replicação desta iniciativa em outras localidades e usinas do país, contribuindo, assim, com o esforço global de mitigação.

No capítulo seguinte, Mello et al. exploram cenários de produção de etanol no Brasil em termos de efeitos da tecnologia flex (bicombustível) no consumo brasileiro de gasolina e o impacto de um maior consumo de etanol sobre às áreas dedicadas à plantação de cana de açúcar destinadas a essa produção. Segundo simulações dos autores, o crescimento do consumo de etanol poderá levar a necessidade de aumento de até $300 \%$ das áreas cultivadas por cana no ano de 2030, correspondendo a 32-39\% do total de área do Estado de São Paulo. Assim, sem uma evolução tecnológica drástica na produção de etanol, o aumento das áreas cultivadas por cana como forma de atender a crescente demanda pelo combustível poderá ter graves impactos sociais e ambientais.

No último artigo da segunda parte, Young et al. discutem proposta para evitar o desmatamento a partir de instrumentos de pagamento por serviços ambientais (PSA). Trata-se de incentivo financeiro destinado a agricultores como forma de manter a floresta "em pé", significando uma fonte adicional de renda para compensar os serviços ecológicos conservados. Todavia, a implementação de ações dessa natureza não são simples e demandam várias circunstâncias para o seu sucesso como, por exemplo, aceitação por parte da comunidade e forte liderança do poder público no processo. Além disso, ainda existem lacunas que precisam ser melhor analisadas para aperfeiçoamento desse mecanismo no Brasil.

A terceira parte do livro trata sobre Adaptação e conta com cinco artigos. No primeiro Murgida e Natezon, ambas da Universidad de Buenos Aires, Argentina, tratam do processo de downscaling social usado para análise de situações de risco em um território específico. O artigo exemplifica de forma sucinta a complexidade de pensar a adaptação à mudança em áreas urbanas, bem como as diferentes forças que podem influenciar o processo. As autoras enfatizam que são necessárias não somente abordagens quantitativas, mas também qualitativas, além de processos de tomada de decisão que devem ser participativos como forma de agregar diferentes percepções da mudança climática e das medidas de adaptação.

No artigo seguinte, Amorim e Pacelli abordam tema importante para discussão sobre mudança climática em centros urbanos debruçando sobre a compreensão das relações e dinâmicas entre urbanização e clima. $\mathrm{O}$ artigo busca discutir como a cidade é responsável por criar um clima urbano e quais suas influencias para a discussão mais recente sobre mudanças climáticas. A partir de uma abordagem escalar, os autores tentam demonstrar que o ambiente construído da cidade é influenciado por condições atmosféricas que alteram fluxos de energia concentrando poluentes e partículas sólidas em suspensão em área que delimita o clima urbano.

Rola et al. analisam a vulnerabilidade de recursos hídricos em áreas rurais e urbanas no Brasil. Os autores analisam diferentes técnicas de uso inteligente de águas das chuvas a partir de dois estudos de caso, sendo um deles referente ao uso de cisternas na região do Semi-árido, nordeste do

Mercator - volume 9, número 18, 2010: jan./abr. 
país; e o outro relativo ao uso de "telhados verdes" em áreas urbanas. Ambas as práticas trazem uma série de benefícios para a população, reduzindo vulnerabilidade e podem ser consideradas práticas autônomas de adaptação, segundo a literatura internacional (PARRY et al., 2007).

O artigo seguinte discute os impactos das emissões de GEE na saúde humana. Neto e Aleixo tomam como ponto de partida uma análise na cidade de Ourinhos. Trata-se de uma cidade média do Estado de São Paulo, com baixa taxa de industrialização e tráfego de automóveis, porém, a queima das plantações de cana dos meses de Abril a Dezembro de cada ano gera uma série de impactos negativos à saúde, sobretudo ao sistema respiratório. Tal prática é realizada durante os meses de clima mais seco, aumentando a concentração de poluentes do ar. O cruzamento dos dados mostra correlação entre doenças respiratórias e a prática da queimada das plantações de cana.

No último artigo da terceira parte, Romero et al. da Universidad de Chile analisam a complexa relação de vulnerabilidade e responsabilidade das cidades em termos de mudanças climáticas. Assim, cidades são comumente tratadas como causa e solução ao aqeuecimento global, uma vez que centros urbanos são grandes emissores de GEE, ao mesmo tempo em que também apresentam oportunidades de inovação e sustentabilidade que podem reduzir consideravelmente as emissões desses gases. Os autores destacam o alto índice de urbanização da América Latina e investigam mudanças de uso do solo em Santiago e Valparaíso, ambas no Chile, e os efeitos em termos de ilhas de calor a partir da análise de imagens de satélite traçando relações entre ocupação do solo, temperatura, poluição, dados demográficos e segregação socioambiental. Os autores destacam que o planejamento urbano e gestão das cidades no país não levam em conta a mudança climática, fato esse que também pode ser estendido para a realidade brasileira.

A última parte do livro trata das conseqüências das mudanças climáticas em sistemas naturais. No primeiro artigo, Neto e Roseghini destacam o processo de ocupação das encostas do litoral norte de São Paulo que, combinados com degradação ambiental, favorecem movimentos de massa e inundações resultantes de chuvas nessa região. A partir da caracterização das variações pluviométricas por meio de série histórica, os autores analisaram por meio de tratamento estatístico o impacto pluviométrico sobre o espaço geográfico da região, identificam a presença de eventos extremos que expõem a população ao risco. No artigo seguinte, Veissid, Instituto Nacional de Pesquisas Espaciais (INPE), descreve experimento para monitorar a mudança climática na América do Sul por meio da análise do albedo, radiação solar refletida pela superfície da Terra e sua atmosfera. Segundo o autor, efeito estufa, temperatura atmosférica, inversão térmica, aerossóis, erupções vulcânicas, entre outros condicionantes, alteram o valor do albedo e tornam-se importantes iniciativas para monitorá-lo como estratégias para melhor compreender as mudanças climáticas.

O último artigo do livro, de autoria de Campos e Wainer, busca apresentar o papel dos oceanos na discussão sobre mudança climática global. Além de duramente impactados pelo aquecimento global, os oceanos são fundamentais para o equilíbrio do clima global contribuindo não só para sua mudança, como também para a variabilidade inerente do sistema climático. $\mathrm{O}$ aumento das temperaturas médias da Terra e o derretimento de geleiras nos pólos deverão levar a uma elevação do nível médios dos mares. Esse aquecimento também terá impactos nos regimes de ventos, chuvas, além das demais interações entre oceanos e atmosfera que poderão desequilibrar ainda mais o sistema climático global.

O livro em questão, além de ser um rico repertório de temas e abordagens teórico-metodológicas, deve ser encarado também como um chamado para maior engajamento das ciências brasileiras em torno do estudo e compreensão da mudança climática e seus impactos no Brasil. Ações governamentais e políticas públicas de enfrentamento do problema requerem um corpo robusto de análises e cenários dos quais o país não dispõem no momento (HOGAN, 2007; NOBRE, 2007; HOGAN; MARANDOLA JR, 2009). Nesse sentido, a geografia assim como as ciências sociais em geral, tem grande contribuição a dar nesse debate. 


\section{Referência Bibliográfica}

ADGER, W.N.; PAAVOLA, J.; HUQ, S.; MACE, M.J. Fairness in Adaptation to Climate Change. Cambridge: MIT Press, 2006.

AYERS, J. International funding to support urban adaptation to climate change. Environment and Urbanization, v.21, n.1, p. 225-240, 2009.

FÜSSEL, H.M. The Risks of Climate Change: A Synthesis of New Scientific Knowledge Since the Finalization of the IPCC Fourth Assessment Report (AR4). Background note to the World Development Report 2010. World Development Report 2010: Development and Climate Change. World Bank, 2008.

GIDDENS, A. The Politics of Climate Change. Cambridge: Polity Press, 2009.

HOGAN, D. J.; MARANDOLA JR, E. (Orgs.). População e mudança climática: Dimensões humanas das mudanças ambientais globais. Campinas: Núcleo de Estudos de População (NEPO/UNICAMP), 2009. HOGAN, D.J. Human dimensions of global environmental change. Ambiente \& Sociedade, v. 10, n. 2 , Jul/Dez., pp. 161-166, 2007.

MARTINS, R.D.A.; FERREIRA, L.C. Assessing the Research on the Human Dimensions of Global Environmental Change in Latin America. Teoria \& Pesquisa, v. 18, n. 2, pp. 31-52, 2009.

MOG, J.; KERSTEN, W.; RAHAMAN, M.M.; KESKINEN, M.; DOWNS, T.J.; WAHID, S.M.; MARTINS, R.D.A.; CONNOR, H.; WILLIAMSON, L.E.; MOEZZI, M.; HOURI, A.; KUMAR, B.; HIREMATH, R.B.; SCHOLTENS, B.; AJAYI, OC; AKINNIFESI, FK.; SILESHI, G.; SOMORIN, O.A. What would be the three key preconditions for jumpstarting or scaling up the transfer of environmentally sound technologies for climate change to developing countries? Natural Resources Forum, v.33, n.4, pp. 334-337, 2009.

NOBRE, C.A. Mudanças Climáticas Globais e o Brasil: por que devemos nos preocupar. Boletim SMET, v. 31, n. 1, Abril, pp. 7-11, 2007.

PARRY, M. et al. Climate Change 2007 - Impacts, Adaptation and Vulnerability. Contribution of Working Group II to the Fourth Assessment Report of the IPCC. Cambridge: Cambridge University Press, 2007.

RAHMSTORF, S.; CAZENAVE, A.; CHURCH, J.A.; HANSEN, J.E.; KEELING, R.F.; PARKER, D.E.; SOMERVILLE, R.C.J. Recent climate observations compared to projections. Science, v. 316, n. 5825, 709, 2007.

RIBEIRO, W.C. Impactos das mudanças climáticas em cidades no Brasil. Parcerias Estratégicas, v.27, p. 297-321, 2008.

SOLOMON, S. et al. Climate Change 2007 - The Physical Science Basis. Contribution of Working Group I to the Fourth Assessment Report of the IPCC. Cambridge: Cambridge University Press, 2007. WILLETT, K.M.; GILLETT, N.P.; JONES, P.D.; THORNE, P.W. Attribution of observed surface humidity changes to human influence. Nature, v. 449, n. 7163, 710-U6.

ZHANG, X.; ZWIERS, F.W.; HEGERL, G.C.; LAMBERT, F.H.; GILLETT, N.P.; SOLOMON, S.; STOTT, P.A.; NOZAWA, T. Detection of human influence on twentieth-century precipitation trends. Nature, v. 448, n. 7152, 461-U4, 2007.

Trabalho enviado em fevereiro de 2010 Trabalho aceito em abril de 2010

Mercator - volume 9, número 18, 2010: jan./abr. 